\title{
LA CONSTRUCCIÓN DE UN NUEVO CAMPO DE CONOCIMIENTO: EL CASO DE LA BIOÉTICA EN VENEZUELA
}

\author{
Enrique Cubero-Castillo ${ }^{1}$
}

Resumen: En este artículo se describe y analiza el proceso de institucionalización de la bioética en Venezuela, en términos tanto de su organización, composición y actividades, como del nivel de gestión pública en el cual se ha institucionalizado. El método empleado es documental, estructurado de manera cronológico-descriptiva, el cual permite establecer relaciones, diferencias, etapas, posiciones y estado actual de los conocimientos en el área de estudio. Se concluye que dicho proceso no ha estado articulado con un programa de formación de capacidades, tanto para el área de investigación como para la gestión.

Palabras clave: bioética, bioética en Venezuela, comités de bioética, institucionalización

\section{Building a new field of knowledge: the case of bioethics in Venezuela}

Abstract: In this article the process of institutionalization of bioethics in Venezuela is described and analyzed, in terms both of its organization, composition and activities and the level of public management in which it has been institutionalized. The method employed is documental, structured in a chronological-descriptive way, which allowed establishing relations, differences, stages, positions and current state of knowledge in the field of study. It was concluded that such process has not been articulated with a program for training capacities, both for research field and management.

Key words: bioethics, bioethics in Venezuela, bioethics committees, institutionalization

\section{A construçáo de um novo campo de conhecimento: o caso da bioética na Venezuela}

Resumo: Neste artigo se descreve e analisa o processo de institucionalização da bioética na Venezuela, em termos tanto de sua organização, composição e atividades, como do nivel de gestão pública no qual foi institucionalizada. O método empregado é documental, estruturado de maneira cronológico-descritiva, que permitiu estabelecer relaçóes, diferenças, etapas, posiçôes e estado atual dos conhecimentos na área de estudo. Se conclui que o dito processo náo esteve articulado com um programa de formação de capacidades, tanto para a área de investigação como para a gestão.

Palavras-chave: bioética, bioética na Venezuela, comitês de bioética, institucionalização

\footnotetext{
${ }^{1}$ Instituto Venezolano de Investigaciones Científicas, IVIC, Venezuela Correspondencia: enrique.cubero@gmail.com
} 


\section{Introducción}

La bioética, desde sus inicios, se desarrolló en dos vertientes: por un lado la bioética clínica, cuya orientación epistemológica tiende a reflexionar sobre los aspectos éticos de la práctica biomédica y la relación médico-paciente, y, por otro, la bioética global, cuyo objetivo principal delineado por Potter se orienta a una reflexión más amplia sobre el uso del conocimiento científico y sus consecuencias para la supervivencia de la vida en el planeta. Ambas orientaciones permanecen en la actualidad como dos tendencias diferentes, aunque no por ello en conflicto.

En este trabajo analizaremos el proceso de institucionalización de la bioética en Venezuela y daremos cuenta de la tendencia que predomina en este país.

\section{Métodos}

Este trabajo, que es de corte documental e histórico, es parte de una investigación más amplia en el cual tuvo como resultado un trabajo de grado de maestría del autor. Se revisó material textual en los que se encuentran: artículos especializados, artículos de divulgación, trabajos monográficos, leyes, normas, códigos, gacetas oficiales y entrevistas publicadas. Por otra parte, se revisó material electrónico, como páginas Web institucionales. La información obtenida se ordenó en una base de datos documental, organizada por fechas de publicación del material, autores y tema principales. Por otra parte, se empleó la técnica de entrevistas en profundidad a actores claves. Todos estos datos permitieron la reconstrucción de la historia.

\section{Resultados}

Al igual que otros países de la región, Venezuela tuvo una disposición de apertura para recibir el paradigma bioético desde los inicios. Puesto que la ética médica había sido uno de los temas recurrentes en nuestro país, impulsada por médicos venezolanos como Luis Razetti(1), en su desempeño en la Academia Nacional de Medicina, y posteriormente por Augusto León, con su publicación de "Ética Médica". Esta reflexión había abonado el terreno para que en el campo de las ciencias médi- cas se prestara mayor atención a este nuevo campo de conocimiento llamado "bioética".

En el marco de las observaciones anteriores nos permitiremos señalar dos periodos de la institucionalización de la bioética en Venezuela, a saber, 1) arribo y puesta en marcha, caracterizado principalmente por la creación de comisiones y grupos interesados en la disciplina y, 2) ampliación, en la cual se adelantan procesos de creación de normativas y códigos de bioética.

\section{1.- Arribo y puesta en marcha de la bioética en Venezuela}

\section{La bioética biomédica}

La bioética en Venezuela(2) ${ }^{2}$ parte del deseo de algunos docentes universitarios, médicos en su mayoría, interesados en la reflexión médico-humanista que se venía dando en otros lugares del mundo. En 1995, Alfredo Castillo Valery, primer médico venezolano con formación en bioética, junto con otros colaboradores e interesados, forman un grupo de trabajo dentro de las instalaciones de la Escuela Experimental de Enfermería de la Universidad Central de Venezuela, que da lugar a la Asociación Civil Comisión Nacional de Bioética CENABI, integrada por el Dr. Augusto León Cechini (presidente honorario), el Dr. Alfredo Castillo Valery (presidente ejecutivo), la Dra. Isis de Landaeta (vicepresidenta), el Dr. Gabriel D’Empaire (secretario), el Dr. Óscar Iván Silva (tesorero) y cinco vocales(3).

Esta comisión, desde su fundación, ha llevado a cabo la tarea de formación de talentos, especialmente en bioética clínica, ofreciendo cursos, seminarios y conferencias, impartidos tanto por conferencistas internacionales como por personal médico venezolano. Además, ha desarrollado una labor importante de revisión de proyectos de investigación en el área médica y asesorías a comisiones de ética locales que han ido surgiendo en nuestro país.

\footnotetext{
2 Véase: Schmidt L. La bioética en Venezuela, primeras huellas. Bioethikos 2007; 1(2): 24 - 38. Este estudio se considera como antecedente a este trabajo, sin embargo creemos que corresponde a un artículo basado en la anécdota propia del autor como participante directo de los acontecimientos y no como un estudio socio histórico basado en fuentes de consulta y trabajos publicados.
} 
En ese mismo año de 1995 se inicia el primer grupo de Bioética Hospitalaria o Comité Asistencial de Bioética, constituido posteriormente como un comité de Bioética para la Investigación, en la institución privada Hospital de Clínicas Caracas; bajo la dirección del Dr. Gabriel D’Empaire, médico Cardiólogo intensivista, con formación en bioética. Dentro de sus funciones se encuentra la revisión de los protocolos de investigación que se realizan dentro de la institución, así como hacer seguimiento a los casos que lo ameriten y promover actividades de formación. Este comité tenía la tarea de revisar y aprobar los protocolos de investigación, en sus aspectos éticos, metodológicos y legales, cuidar que se ajustaran a las normas internacionales vigentes para la investigación biomédica y lo referente a la ley de ejercicio de la medicina en Venezuela, así como atestiguar que cumplieran con los principios de la bioética.

Para ello, dicho comité organizó un reglamento interno, en el cual se establecían las normas de trabajo y funcionamiento para la toma de decisiones en casos complejos, y un instructivo para los investigadores médicos. Creó además formatos para la obtención de los requerimientos necesarios para someter un protocolo a la consideración del comité. Sin embargo, debido al desconocimiento que entre el gremio médico se tenía de esta disciplina, las reacciones y dificultades no tardaron en aparecer, percibiendo al comité como una instancia entorpecedora de la libertad para la investigación. Por otra parte, la carencia de personas formadas en el área y la falta de legislación en bioética en nuestro país, hacía más difícil la evaluación de los casos(4).

Este comité se mantiene en la actualidad y se considera una experiencia exitosa de organización de un comité de bioética - a pesar de que para el momento de su creación no estaba claro cómo este debía funcionar - que posee una amplitud de funciones, entre ellas: función docente, con los programas dirigidos a la formación de talentos; función consultiva, con los análisis y deliberación de casos concretos de investigación y, función divulgativa, con la difusión del conocimiento y experiencia de la actividad del comité(4).

\section{El papel del Consejo Nacional de Investigacio- nes Cientificas y Tecnológicas (CONICIT) de Venezuela}

La bioética en el CONICIT (en la actualidad Fondo Nacional de Ciencia y Tecnología, FONACIT) se inicia a partir de la Conferencia Científica de la VII Cumbre Iberoamericana de jefes de Estados y de Gobiernos, realizada en Caraballeda, Estado Vargas en el año 1997, la cual concluye con esta recomendación:

"Se recomienda la creación de Comisiones $\mathrm{Na}$ cionales de la Ética que amplíen y profundicen el desarrollo de los principios básicos y promuevan la adopción de códigos complementarios, que hagan posible una actividad científica y tecnológica con apego a elementales principios éticos y morales. Todo esto según las condiciones particulares de cada país y en coordinación con la Comisión Internacional de Ética de las Ciencias, creada por la UNESCO"(5:197).

El CONICIT, para dar respuesta a la recomendación y adherirse a las convocatorias de los organismos internacionales, impulsa, a través del directorio de la institución y estos, a su vez, delegando la parte operativa en la socióloga Eva Briceño, llevar a cabo la organización de la Comisión de Bioética y realizar mesas de trabajo para la redacción del Código de Bioética y Bioseguridad ${ }^{3}$, que finalmente fue publicado en 1999.

La creación del Código se caracterizó por ser un proceso lento y con secciones de trabajo arduo. $\mathrm{La}$ primera parte de la organización de la Comisión estaba destinada a convocar a diversos expertos venezolanos dispuestos a colaborar con la elaboración y redacción de los artículos que formarían parte del Código, y estos debían ser expertos no solo en sus áreas específicas, sino también con una base de formación en ética o, por lo menos, que estuvieran dispuestos a formarse en la materia.

La Comisión de Bioética y Bioseguridad queda establecida por un equipo de trabajo conformado por doce personas capacitadas para la evaluación de los proyectos, constituida de la siguiente manera: investigadores y profesionales, para formar un equipo de investigación interdisciplinario, un representante del clero o filósofo con experticia en

${ }^{3}$ Datos obtenidos a través de entrevista a actor clave. 
el área y un representante de la comunidad con experticia en derechos de los animales. La tarea principal, como expresa el mismo documento, es evaluar los proyectos de "biomedicina, los involucrados en manejo de Organismos Genéticamente Modificados, agentes biológicos peligrosos y organismos exóticos" (6).

El propósito de este primer Código era servir de orientación normativa para la elaboración de los proyectos de investigación científica que se realizan en el país. Sin embargo, su objetivo no pretendía ser en ningún momento punitivo, sino orientador de las acciones que los científicos, responsablemente y de acuerdo con su conciencia, llevan a cabo en sus investigaciones. Asimismo, se establecía que la revisión del código debía hacerse cada cuatro años, con la intención de actualizar los campos de investigación y las perspectivas normativas de acuerdo con los avances de la ciencia.

La tarea llevada a cabo por el CONICIT fue de gran importancia, puesto que se estableció una base normativa anclada en realidades concretas de nuestro país, con expertos y profesionales que ejercían la actividad de investigación y que tenían la experiencia para determinar cuáles eran los nudos críticos y comunes a los cuales el código debía ofrecer salidas oportunas.

Esta tarea se ha venido realizando de acuerdo a lo establecido, logrando hacerse la primera revisión en 2003, y la actual en 2008, respectivamente. Aunque la estructura inicial ha cambiado con el tiempo, estableciendo nuevos criterios de evaluación y nuevas áreas de investigación, el espíritu de la norma permanece vigente y sigue cumpliendo con el objetivo orientador que se propuso. Sin embargo, la actividad llevada a cabo por la comisión de bioética y bioseguridad del actual organismo, es poco conocida y reducida en su radio de acción. Por lo cual, es necesario que en adelante sus esfuerzos estén orientados a la promoción, formación y divulgación de las implicaciones éticas inherentes a la actividad de investigación que se realiza en el país, con especial énfasis en la generación de espacios de debate y participación pública y ciudadana, en los que se discutan las oportunidades y riesgos que implica la aceptación y patrocinio de los proyectos de investigación llevados a cabo en el país.

\section{Comisión Nacional de Bioética del Ministerio del Poder Popular para la Salud}

En este proceso de institucionalización que se realizaba en al país, surgió otra propuesta institucional desde el Estado, con el nombre de Comisión Nacional de Bioética, aprobada oficialmente mediante Gaceta Oficial N 307.095 del 8 de octubre de 1998. Esta comisión se crea con carácter permanente con el objetivo de regular y supervisar todo lo relacionado con los aspectos éticos de la investigación y atención en salud.

La comisión queda oficialmente conformada por diez personas, encabezada por el ministro y demás representantes del Ministerio de Salud, así como de un representante de los entes adscritos a dicho gabinete con competencia en la materia. Dentro de sus funciones específicas se encuentra la vigilancia y supervisión de lo relacionado con los aspectos éticos de la investigación, elaboración de normas para la creación de comités de ética en los hospitales, la promoción de actividades formativas y la asesoría al ministro de Sanidad en la materia y objeto de la comisión.

Legalmente, esta iniciativa institucional tuvo vigencia hasta 2010. Sin embargo, enseguida de haberse creado, hubo cambios de autoridades en dicho ministerio y no se continuó con el desarrollo de la idea inicial sino hasta 2010, en que se retoma la idea de una Comisión de Bioética y Bioseguridad, con los objetivos de investigación y promoción de los aspectos éticos en el área de la salud, que permanece vigente.

\section{Comisión de Bioética en el Instituto Venezola- no de Investigaciones Cientificas, IVIC}

A casi 40 años de funcionamiento, el Instituto Venezolano de Investigaciones Científicas, IVIC, crea una Comisión de Bioética, acordada por el consejo directivo de la institución, en su sección ordinaria 1016 del 16 de diciembre de 1998, bajo la resolución $\mathrm{N}^{\circ} 48$. Los motivos que dieron pie a esta decisión fueron que la investigación indiscriminada, que no cumple con los presupuestos éticos, puede acarrear graves perjuicios personales y sociales, y que además la ciencia y la tecnología, que avanzaba con gran rapidez, hace aparecer nuevos problemas éticos que, en ocasiones, 
sobrepasan a los investigadores. De manera que, con la creación de la comisión de expertos que evaluara los proyectos, los investigadores podían realizar sus actividades con base en valores éticos, en correspondencia con la legislación nacional e internacional que rige la materia.

Se constituye entonces dicha comisión, como un órgano asesor y de control del consejo directivo del IVIC, para velar por el cumplimiento de lo establecido y con total competencia para revisar los protocolos de investigación y todas las actividades que involucran experimentación con seres humanos. En la resolución antes mencionada, queda establecido que el número de participantes de dicha comisión no puede ser inferior a siete, integrado por profesionales de diversas áreas de la ciencia, incluyendo por lo menos un miembro externo al Instituto(7). De esta forma se busca garantizar que la comisión quede integrada por un equipo multidisciplinario que responda debidamente a la evaluación de los proyectos.

Esta comisión -aun cuando se mantiene vigente- es poco conocida, incluso dentro de la institución. Su actividad se ha encargado de la revisión de los proyectos de investigación de los estudiantes que se forman en los posgrados, y pocas veces de los investigadores. Una razón probable de esta situación puede estar relacionada con el hecho de que estos últimos envían directamente sus proyectos a la Comisión de Bioética y Bioseguridad del FONACIT, ya que es requisito contar con su visto bueno para acceder a subvenciones del fondo. Otra razón puede estar relacionado con la ausencia de normas claras que regulen el funcionamiento de la distintas comisiones de bioética que existen en el país, por lo que queda a criterio de los investigadores el envío de sus proyectos de investigación si se les exige.

Lo anteriormente descrito queda evidenciado en un documento enviado por la Comisión de Bioética del Instituto, a solicitud del Ministerio de Ciencia y Tecnología en 2002, como lo señala Apitz(8): "En nuestra experiencia, el aspecto más importante se refiere a la estandarización del funcionamiento de los diversos comités que deben existir en el país. Para eso creemos que el primer paso debe ser la creación de un Consejo Nacional de Bioética"; dicha recomendación no prosperó en el Ministerio de Ciencia y Tecnología.

En el IVIC una nueva Comisión de Bioética se crea en 2009, según la resolución $\mathrm{N}^{\circ} 127$ del consejo directivo, con el objetivo de velar porque las actividades de investigación en las que participa el instituto y que requiera el uso de animales de experimentación y animales silvestres cumplan con la legislación venezolana y con valores éticos universales.

Ambas comisiones, que hasta ahora funcionan en el instituto, están dispuestas como órganos asesores del consejo directivo y, en la práctica, funcionan como compartimentos estancos dentro de la institución.

Hasta este momento de nuestro análisis consideramos que termina el periodo denominado "de arribo y puesta en marcha" de lo que constituye este proceso de institucionalización. En efecto, lo que a continuación se describe se refiere a las normativas legales vigentes, el comienzo de un programa de formación de maestría en bioética clínica, la creación de una revista especializada en el campo de la bioética y las distintas restructuraciones de las comisiones antes descritas.

\section{2.- Proceso de Ampliación de la Bioética en Ve- nezuela}

El proceso de ampliación corresponde con los distintos esfuerzos que a lo largo de estos años han ido sentando las bases del paradigma bioético en nuestro país. Este proceso se ha caracterizado por acciones concretas que dan cuenta de nuevas formas institucionales para desarrollar la disciplina. La creación de normativas legales que parten de un hecho importante en nuestro país, como es la Reforma Constitucional de 1999, la modificación de la Ley de Ciencia y Tecnología de 2001, la nueva perspectiva introducida en el Código de Bioética y Bioseguridad del FONACIT, entre otras.

\section{Bioética en la Constitución de la República Bo- livariana de Venezuela de 1999}

Con la llegada de Hugo Chávez a la presidencia de la República, mediante la consulta popular es convocada una Asamblea Nacional Constitu- 
yente, en la que participan todos los sectores representativos de la vida nacional y en la cual se aprueba reformar la Constitución Nacional de 1961. Con ello se introduce por primera vez en la nueva constitución el respeto por los principios bioéticos, específicamente en el capítulo VIII: De los derechos de los Pueblos Indígenas, en el artículo 122, en el que se establece que el Estado debe reconocer la medicina tradicional con "sujeción a principios bioéticos", y en el capítulo IX: De los derechos Ambientales, en el artículo 127, donde se establece que la ley que se refiera a principios bioéticos regulará lo concerniente a la protección del ambiente, la diversidad biológica, los recursos genéticos y la prohibición de no patentar el genoma de los seres vivos.

Con este paso importante se reconoce la vinculación entre los problemas bioéticos y el Derecho, y se amplía la reflexión hacia lo jurídico y social. Con la introducción de este aspecto en la constitución de nuestro país se abre la posibilidad de legislar al respecto, y con más pertinencia a las particularidades propias de la realidad venezolana.

\section{El Código de Ética para la vida del FONACIT}

Desde la primera redacción del Código, en 1999, hasta la última edición de 2008, se han producido cambios significativos tanto en la forma y estructura del contenido, como en el fondo del mismo. El primer documento redactado se dividía en dos partes amplias, (biomedicina y Organismos Genéticamente Modificados, agentes biológicos peligrosos y organismos exóticos) y finalmente en la norma de funcionamiento de la comisión(9).

La segunda edición de 2002 presenta tres partes: 1) principios de la bioética y bioseguridad, 2) normas de bioética y bioseguridad para la investigación con organismos vivos, ambiente y bioterios, el cual se subdivide en seis capítulos, y 3) normas de la Comisión de Bioética con anexos del consentimiento informado previo y las normas Unesco para las comisiones de bioética.

La tercera edición, presentada en 2008, presenta una modificación importante y es el cambio de nombre por el de Comisión de Ética, Bioética y Biodiversidad, en concordancia con lo establecido en la Ley Orgánica de Ciencia, Tecnología e
Innovación, 2001, aunque su estructura interna permanece igual a la edición anterior.

\section{Ley Orgánica de Ciencia, Tecnología e Innova- ción, LOCTI, 2010}

La LOCTI, cuyo objetivo es organizar el Sistema Nacional de Ciencia y Tecnología, así como servir de orientación para establecer los lineamientos de las actividades y políticas de investigación, financiamiento y apropiación social del conocimiento científico y tecnológico, no deja por fuera lo correspondiente a la bioética, sino que lo establece como un requisito importante para que la realización de las actividades de ciencia, tecnología e innovación estén debidamente encaminadas al respeto de los derechos humanos y los acuerdos internacionales suscritos por nuestro país.

En el artículo 5 se destaca que: "Las actividades de ciencia, tecnología e innovación, y la utilización de los resultados deben estar encaminadas a contribuir con el bienestar de la humanidad, la reducción de la pobreza, el respeto a la dignidad y los derechos humanos, y la preservación del ambiente" (10), pasando por la ética, probidad y buena fe, los principios bioéticos, las comisiones de ética, bioética y biodiversidad, y finalmente la protección de los conocimientos tradicionales.

La LOCTI fue modificada y aprobada en 2010, y lo descrito fue objeto de una nueva concepción más amplia de los conceptos de ética para la ciencia, la tecnología y la innovación. En esta nueva concepción, la bioética, que aparecía como saber especifico, fue modificada por el concepto de "ética para la vida”, que responde a parámetros más amplios de consideración del concepto de vida como un todo. Es decir, está basado en principios de equidad, justicia y solidaridad, tanto entre los hombres que ahora habitamos el planeta como en las generaciones futuras, de manera que, partiendo de la complejidad del sistema ambiental del cual formamos parte, nos vemos obligados éticamente a mantener relaciones equilibradas entre la generación de conocimiento y el uso que se haga del mismo.

Es importante señalar que la aprobación de la Ley de Diversidad Biológica en 2000, tiene como finalidad establecer los principios rectores de la 
conservación de la diversidad biológica en nuestro país, tomando en consideración los aspectos éticos por la utilización de la diversidad biológica, la cual debe realizarse en conformidad con los principios establecidos por la bioética

\section{Universidad Central de Venezuela (UCV), Co- misiones de Bioética y Maestría en Bioética Clínica}

$\mathrm{Al}$ igual que las demás instituciones mencionadas, en las cuales se han constituido comisiones de bioética, la UCV ha sido un escenario en el cual han proliferado distintas comisiones que funcionan de manera separada. Muchas responden a intereses particulares, de acuerdo con los lineamientos y actividades de los institutos y escuelas que las conforman. El Hospital Clínico Universitario, el Instituto de Biomedicina, el Instituto de Higiene Rafael Rangel, Facultad de Odontología, Facultad de Ciencias, son algunas de la instancias universitarias donde se cuenta con un comité de bioética.

Con el impulso y la experiencia del CENABI, se da inicio a la primera Maestría en Bioética en nuestro país, particularmente para fomentar la bioética clínica y el estudio de los problemas éticos surgidos del desarrollo de la tecnología biomédica. Entre los temas de estudio que son tratados se encuentran los relacionados con el comienzo y final de la vida humana y con la calidad de vida, promoviendo la ética de la investigación biomédica.

\section{Revista Latinoamericana de Bioética}

Esta iniciativa parte del interés de nueve organizaciones nacionales de bioética de América Latina, y de un representante de la Universidad Complutense de Madrid, reunidos en la Universidad de Valencia, Venezuela, quienes asumieron el compromiso de crear esta revista virtual. Y ello porque, en este siglo de la ciencia, la comunicación y la participación femenina, el planeta, el mundo y los países latinoamericanos continúan experimentando la constante agresión a la naturaleza y a los derechos humanos, con el imperio de la injusticia y la inequidad.

\section{La Revista Virtual Latinoamericana de Bioética,}

desde 2008, aspira a cumplir el compromiso de mantener un espacio de reflexión y difusión conceptual de los valores éticos y morales, del respeto a los derechos humanos y al bienestar de la convivencia humana y de su equilibrio armónico con la integridad de la naturaleza, al mismo tiempo que recoja y divulgue los productos de su investigación y las consecuencias de la integración de su estudio en todos los niveles de educación. Fue fundada por Oswaldo Chaves Ceballos (19292011) y aparece con una periodicidad semestral, indizada en Latindex bajo el No 19850.

\section{Un nuevo centro para la investigación. El La- boratorio de Bioética y Biopolítica del IVIC}

El IVIC es uno de los centros de investigación del país que ha logrado posicionarse a nivel internacional como un centro de referencia. Dentro de la estructura de la institución se desarrollan diversos campos de investigación que van desde la ciencias básicas y aplicadas hasta las ciencias sociales. Uno de los centros dedicados a las ciencias sociales es el Centro de Estudios de Transformaciones Sociales, Ciencia y Conocimientos, (antiguo Centro de Estudios de la Ciencia), que se compone de tres laboratorios: 1) Laboratorio de Estudios de la Ciencia, Tecnología y Conocimientos, cuyo objetivo principal es el estudio de la naturaleza y dinámicas de las prácticas científicas, tecnológicas, de innovación y de otras formas de conocimiento, tanto como sus transformaciones, interrelaciones e impacto en la sociedad; 2) Laboratorio de Estudios del Espacio Público, abocado a la investigación sobre las formas de representación, deliberación y participación en el espacio público, como ámbito de interacción, intercomunicación y transformación de la sociedad; y 3) Laboratorio de Bioética y Biopolítica, dedicado a la investigación a partir de un enfoque integral y transdisciplinario centrado en la dimensión epistemológica de la bioética y la biopolítica, incluyendo sus dimensiones práctica y normativa.

En este espacio se asume la bioética y la biopolítica como dos áreas complementarias, entendidas como la reflexión y acción sobre principios, normas y valores de las prácticas que afectan a la vida y del ejercicio del poder sobre la misma.

\section{Consideraciones finales}


A manera de conclusión, podemos decir que la bioética en Venezuela se encuentra aún en una etapa de asimilación, desde la cual es necesaria avanzar con el fin de superar la bifurcación originada desde el nacimiento del paradigma bioético y reorientar la reflexión hacía una bioética global, capaz de entender al hombre como parte de un sistema planetario complejo. El proceso de institucionalización descrito ha tenido un carácter lento y circunscrito, no articulado a un programa de formación de capacidades en el área, debido a que los pocos núcleos de acción creados no guardaron estructuración y los esfuerzos realizados fueron particulares y fragmentarios.

La constitución y dirección de los comités de bioética en Venezuela han sido producto de intereses particulares, superponiendo el espacio privado sobre el público en la gestión y administración de la investigación; y el proceso de institucionalización que hasta el momento se ha llevado a cabo, permanece anclado en presupuestos epistemológicos foráneos, desarticulados de las políticas públicas actuales de investigación.

De manera que es necesario abrir nuevos espacios para la formación y la investigación en bioética, con el fin de reflexionar sobre el impacto de la ciencia y la tecnología a partir de nuestras visiones históricas y culturales. Y que dicha investigación en bioética trascienda desde la visión práctica (comités de bioética) y normativa (códigos de bioética) a una dimensión epistemológica contextualizada, para que se constituya en una verdadera fuerza biopolítica que haga posible el establecimiento y control de políticas de investigación efectivas, que ayuden a resolver los problemas urgentes de nuestro país.

\section{Referencias}

1. Cubero-Castillo E. La ética médica en Venezuela (1895-1912). Bitácora-e, Revista Electrónica Latinoamericana de Estudios Sociales, Históricos y Culturales de la Ciencia y la Tecnología 2012; 1. Disponible en: http://www.saber.ula.ve/ handle/123456789/35555 Consultada 25 de febrero de 2015.

2. Schmidt L. La bioética en Venezuela, primeras huellas. Bioethikos 2007; 1(2): 24-38.

3. Castillo A. Lista de Cursos de Ampliación en Bioética, dictados por el Centro Nacional de Bioética (CENABI). Boletín Informativo de la Comisión de Estudios de Postgrado de la Facultad de Medicina UCV 2010; 13(1).

4. D’Empaire G, D’Empaire M. Comité de Ética Hospitalaria. Primera Experiencia Nacional. Clínica Médica HCC 1999; 4-23.

5. CONICIT EN CUENTAS. Hacia el Código Nacional de Bioética, № 7. Caracas, abril 1997.

6. CONICIT. Código de bioética y bioseguridad. Táchira: Edición Fundacite; 1999.

7. Instituto Venezolano de Investigaciones Científicas, IVIC. Resolución N48, del 21/12/1998. IVIC; 1998.

8. Apitz R. Comités de Bioética. Gaceta Médica de Caracas 2002; 110(1): 110-113.

9. FONACIT. Código de Bioética y Bioseguridad. Segunda y tercera ediciones, 2003 y 2008; Fonacit; 2008.

10. República Bolivariana de Venezuela, Ministerio del Poder Popular para Ciencia, Tecnología e Industrias Intermedias. Ley Orgánica de Ciencia Tecnología e Innovación 2001. Disponible en http://www.ivic.gob.ve/varios/locti.pdf. Consultado 25 de febrero de 2015 .

Recibido: 28 de mayo de 2013

Aceptado: 25 de julio de 2013 